\title{
4
}

\section{Automating Labour and the Spatial Politics of Data Centre Technologies}

\author{
Brett Neilson and Ned Rossiter
}

Without data centres, automation stops. Data centres are communication infrastructures that store, process and transmit digitally encoded data. They offer cloud-based services to clients that include the automation of organisational routines such as workflow processes and systems management. Promoted as optimising efficiencies, data centres automate task-oriented work while also impacting on labour practices in ways that calibrate work to computational activity. One of the key functions of data centres over the coming decades will consist in supporting automated economies with the integration of artificial intelligence, machine learning and robotics into processes of capital valorisation and accumulation. Stemming from a project entitled "Data Farms: Circuits, Labour, Territory" (http://datafarms.org) that investigates the expansion of the

\footnotetext{
B. Neilson · N. Rossiter $(\bowtie)$

Institute for Culture and Society, Western Sydney University, Sydney, NSW, Australia

e-mail: n.rossiter@westernsydney.edu.au
} 
data centre industry in Asia, this chapter engages the spatial politics of data infrastructures in the age of automation. We focus on data centres in Singapore, which is renowned as a growth hub for data storage and processing installations. Because data centres are themselves automated environments and provide infrastructure that enables automation in workplaces located in diverse and often physically distant sites, they offer a strategic object for research on the uneven and varied implications of automation for labour.

In this chapter, we tackle the nexus of automation and labour by investigating Singapore's data centre industry across diverse milieus. These include the historical legacy of colonialism in Southeast Asia, the continuities between data extraction enabled by data centres and other extractive practices, the capacity for automated technologies to acquire sovereign-like powers, the aesthetic dimensions of data's relations to natural and artificial environments, and the significance of automation for state transformation. Our wide-ranging approach reflects the variety of ways in which data centres recast automation's consequences for labour. Although the issue of whether automation displaces jobs or moves them across sectors is part of our analysis, we do not seek to resolve this age-old question that generates both fantasies and fears about the eradication of work by machines. Rather, we approach the human-machine nexus from diverse angles to suggest that the bond of automation and labour has broader relevance for changing relations between space, economy and politics. In this regard, we understand data centres not merely as technical infrastructures but also as political institutions, which shift power relations across wide spatial vistas and contribute to changing patterns of geopolitics and governance across diverse geographical scales.

Data centres lend an infrastructural dimension to labour politics. The material qualities of data centres - which include hardware and software capabilities along with the architectural form of the buildings, environmental impact of operations and geographical settings-become evident when political strategy and organising approach these digital infrastructures as relevant to understanding labour's situation in changing economic circumstances. Our analysis expands the debate on automation 
and labour to encompass issues of media, extraction, standards, geopolitics, territory and infrastructure. We ask how the division of labour across the spectrum of data servicing industries generates spatial formations that standard accounts of political economy in the tech industry struggle to explain. Focusing on Singapore's data centres and their positioning within wider vistas of capitalist activity and institutional transformation gives a geopolitical anchor to our investigation of how labour transitions to a society of automation.

How can we understand the variation of labour within the footprints generated by data centres when automation threatens to displace the human from the machinery of capital? Answering this question in the context of data centres in Singapore means positioning these installations in relation to other forms of digital infrastructure as well as considering their interaction with forms of political power vested in the state. Our approach combines an empirical concern for the technical operations of data centres with an investigation of the material effects of these operations at sites where labour forces interact with client machines that connect remotely to data centres by means of network topologies that take infrastructural form in cabling technologies and data transfer protocols. We question zero sum narratives of automation and job loss in an analytical frame at once attentive to local conditions and the wider spatial and temporal transformations of digital capitalism.

The history of communications media and transport technologies shows how space and time are endowed with tendencies and propensities specific to the material properties of these systems (Innis, 1951). Similarly, infrastructural facilities such as data centres generate spatialities and temporalities coincident with the operational logic of machines. Such a feature runs against the grain of conventional understandings in international relations and area studies of space as the object of an inter-state contest between civilisational cultures. Temporal features such as low-latency, speed, switching and computational decision-making are likewise antithetical to modern tropes of time as linear, progressive and evolutionary. Machine time and infrastructural space have implications for the performance and experience of work in ways that inform our analysis of labour regimes in contemporary capitalism. 
Automated technologies operating from data centres reconfigure key industries, including transport and logistics, manufacturing, education and training, health and medical services, finance and banking. However, because the data economy extends beyond industry as narrowly conceived, we also consider the labour of data production as it applies to the use of social media and other digital services. These services, which expand labour socially beyond the confines of industry, generate large datasets by drawing upon forms of social cooperation often pursued for pleasure or convenience. Indeed, data harvested in this way can provide a basis for training automated technologies. They thus supply a resource for firms that seek to profit by developing and renting out artificial intelligence capacities. We draw attention to the material infrastructures that enable such data extraction (Couldry \& Mejias, 2019; Mezzadra \& Neilson, 2017).

Our inquiry is geopolitical in its focus on Singapore, an important data centre hub where both Chinese and US tech companies are active. Aside from the question of how Singapore's data centres establish territorial reach that crosses and reconfigures the Southeast Asian region, the competition between Chinese and US tech firms is evident in this context. The ownership and control of standards is an aspect of this competition. We argue that the tussle to institute and spread standards for data transfer and artificial intelligence registers a mode of power that shifts relations between businesses and states. Accordingly, we position Singapore as a laboratory for geopolitical entities intent on expanding their power and influence across regional and global scales.

\section{New Dreams-Old Nightmares}

Since its separation from Malaysia in 1965, Singapore's economic prosperity has been built on subjecting state capitalism to market competition and attracting foreign investment, initially in the manufacturing and petroleum industries and then in more capital intensive sectors such as technology. Starting in the mid-2000s, the government has supported the island's emergence as a data centre hub by offering foreign tech companies attractive tax rates, flexible labour arrangements, ready 
electricity and water supplies and lucrative research and development opportunities. Singapore now hosts around seventy state-of-the-art data centres, attracting approximately 50 per cent of the market share in Southeast Asia (BroadGroup, 2016) and positioning it to benefit from artificial intelligence and machine-learning technologies.

Data centres in Singapore establish infrastructural connections with territorial reach that extends across and reconfigures the Southeast Asian region. To enter one of these installations is to encounter a sterile and securitised environment. Flashing lights and humming fans register the presence of workforces that occupy the client end of network architectures that connect data centres to the outside world. Server load indexes distant labour. Whenever a firm locates servers in a data centre or hires services from a cloud provider, its workforce begins to interact with machines whose location may be unknown to them. This creates infrastructural relations and business possibilities between otherwise disconnected labour forces. For instance, a securely employed office worker in Malaysia and a precarious data entry worker in Indonesia may interact with servers in the same Singapore installation, or even use services delivered by the same physical machine. Because data centres obscure connections between parties that sit on the client end of server/client architectures, relations of this kind among labour forces are not evident to those without inside knowledge about the network topologies and client list of data centres. The world of business enters into a kind of transactional promiscuity not foreseen in strategic planning documents. Such operational obscurity enables data centre companies to recommend business relations of potential interest to their clients, opening additional revenue streams for data facilities and further consolidating their capacity to make worlds.

The example of workers in Indonesia and Malaysia interacting with servers in the same Singapore facility gives an idea of how data centres create connections across the global division of labour, crossing differences of space, gender, race, citizenship, occupation and employment status and social identity. To understand more fully the extent and implications of these connections, we must register how interaction with servers in data centres supports the development of machine learning and 
other artificial intelligence technologies. Data extracted from the activities of workers and users of digital services such as social media platforms provide the raw material from which tech companies build automated services. This situation means that the jobs of workers who interact with servers in data centres are increasingly at risk of elimination through automation. The keystrokes and other inputs provided by these workers provide data that can potentially train artificial intelligence to perform the same tasks. The current wave of fear surrounding the automation of labour extends not only to manufacturing jobs that new-generation robots might perform. Although such displacement is a growing concern in Southeast Asia (Chang \& Hunyh, 2016), the jobs of service, mental or immaterial labourers who interact with digital technologies are also at stake.

New dreams of artificial intelligence inspire old nightmares of labour loss. In recent years, a raft of news reports and policy documents have delivered fearful prognoses of job displacement due to automation (see e.g., Frey \& Osborne, 2013; Méda, 2016; Regalado, 2012). A prominent claim in these reports and documents is that these jobs will not shift to the science and technology sectors that drive technological change, as supposedly has been the case in previous waves of automation. In other words, the structural ascent of workers into high-level tertiary industries and economies has hit a peak over the last thirty years and is now facing gradual termination. A contrasting response from feminism and science and technology studies envisions the reabsorption of work by other means, insisting that futures are always social rather than driven by markets and technologies (see e.g., Wajcman, 2017). Another prevalent argument stems from versions of accelerationism that imagine an elimination of work and a repurposing of modes of production to invent post-capitalist futures (Srnicek \& Williams, 2016).

Overall, the debate is stuck between arguments about job loss that repeat positions on mechanisation and labour derived from early political economy and claims for the powers of automation based on technical knowledge of code, algorithms, neural networks and other relevant programming techniques. We seek to move beyond this impasse by placing the question of automation and labour in the context of the data storage and processing facilities that allow artificial intelligence and 
machine learning to proceed. Our argument has affinities with that of Benanav (2019, p. 121), who contends that automation technologies may lead to an under-demand for labour but that the primary reason for this waning of labour demand is "the absence of a corresponding pace of job creation in the wider economy". In highlighting the dependency relation between labour, automation technologies and data regimes specific to the operational logic of data centres, we seek to examine how this situation shifts unevenly across spatial variegations in the global economy that are increasingly shaped by routines and topologies of digital networking.

\section{$2 \quad$ Racking and Stacking}

Automation has a long history. From the jacquard loom to the centrifugal governor, the Taylorist production line to just-in-time logistical systems, automation has tied industrial modernity to the experience and conditions of labour. Automation has functioned as a connecting device across racial and gendered divisions of labour. To what extent do the relations between automation and labour shift with technologies of artificial intelligence and machine learning? A familiar claim is that earlier techniques of automation used data to perform linear and repetitive tasks whereas contemporary artificial intelligence allows pattern recognition and learning that can mimic human behaviour and thus replace or augment mental labour tasks (Miller, 2018). Beatrice Fazi (2018) argues that artificial intelligence is alien to human thought and thus the relation between automation and labour is not one of simulation. Whatever the limits of machine learning and artificial intelligence, their political and economic significance cannot be underestimated, particularly when services based on them increasingly inform the business models of tech firms spearheading research and development in this field. Ideology and cultural values influence technology design, and therefore hold a shaping force on the experiences and position of people who interact with automated technologies. Business models, in other words, are not merely fanciful discourses, vision statements and marketing campaigns divorced from the social world in which they circulate. 
The extraction and aggregation of data is a precondition for contemporary artificial intelligence and machine learning. Systems complexity can only evolve from the volume and quality of data available for these techniques to crunch. Such an operational requirement situates the data centre as the optimal storage facility and repository for artificial intelligence and machine learning. There is also a political economy at work here. The commercial ownership of data centres frequently does not coincide with the ownership of data trafficking through such installations. Data centres store, process and transmit data whose provenance potentially stretches across many quite different firms and clients. These installations can occupy a meta-operational role by drawing on and accessing a substantial corpus of data for artificial intelligence and machine learning purposes, developing commercial applications on this basis. Storage, transmission and processing, in other words, are just one dimension of the business model of data centres, which are poised to expand services and capacity in the realm of computational cognition.

Advances in machine learning algorithms are central to optimising data centre operations. Some of these applications address problems of energy efficiency and long-term environmental prediction or the optimisation of computational processing power through the development of neural networks and cloud delivery services. However, the automation of labour in data centres is also on the frontier of developments in machine learning. Take the example of data centre jobs at Google, just to name one prominent operator in the Singapore environment. While data centre jobs at Google have expanded tenfold over the past decade, employment rates are now on the decline. The human is now effectively interchangeable with the machine: "The ability to pull together disparate bits of information", as one Google executive explains. As this executive goes on to say: "The cut-and-dry division of labour, where some people did racking and stacking, some provisioned servers and installed operating systems, some oversaw network connections and others did service maintenance in production, has been replaced by automation" (Sverdlik, 2017, n.p.).

As far as data centre management goes, functions usually divide between infrastructure and business management. The former has itself become a profuse field of automation with the introduction of "data 
centre infrastructure management" (DCIM) software in a range of corporate packages with names like Sunbird, Nlyte and Tuangru. These packages bridge information across organisational domains, including data centre operations, facilities and information technology to configure workflows, power use and the like. We can think of them as kind of a meta-automation that works across different datasets and infrastructural elements to make routine automation possible. DCIM packages also enable remote operation of data centres, meaning that management can push staff on the ground to a minimum-a minimum that nonetheless is usually gendered male.

The high degree of automation in data centres does not correspond to the making of a business environment where availability of personnel presents no challenge. A French national working for a major tech firm in Singapore told us: "The more you go to the north of Asia, the more arms and legs there are running the things". He referred to Malaysian government efforts to attract data centres with real estate costs, electricity tariffs and water availability. While finding personnel to perform routine tasks in data centres poses no problem, the availability of skilled individuals to intervene at the level of infrastructure management presents difficulties. Cultural variations are relevant, for instance, in running data centres in Thailand, the Philippines or Japan. According to our informant, if companies are building "solutions" to address the local market, they need to hire local players. If they are trying to go beyond the local market, they need technical and management personnel to align with the culture of the client, meaning that more cosmopolitan and outward-looking attitudes become a component of employability. As a consequence, in-house expertise is distributed according to the cultural variation of markets and client needs. Automation does not simply subtract labour from capital, in other words, but rather multiplies the geocultural settings that supply data capitalism with knowledge that services machine operations. This multiplication and supply involves people as much as machines, and political terrains of population and culture as much as digitally networked topologies of communication.

Whatever the significance of automation for labour within data centres, it is at the client end of the architectures that extend from these installations that the nexus of labour and automation is most intense. We 
have already discussed how users and workers who interact with client machines that connect to data centres furnish data that can train algorithms to perform their jobs, or at least tasks that are part of their jobs. In the case of data centres in Singapore, this possibility extends across wide regional vistas due to the extensive network capabilities of these installations. To understand how server-client architectures extend the net of automation beyond the data centre, we must account for the way in which these architectures interact with technologies of virtualisation within the data centre.

In the contemporary data centre, east-west traffic (between servers in the same facility) exceeds north-south traffic (between servers and clients beyond the data centre). Different topologies with names like Clos, fat-tree, Dcell, BCube, c-Through, Helois, PortLand and Hedera physically connect servers to each other within data centres (Liu et al., 2013, pp. 7-23). Each of these configurations has its own purpose and implies trade-offs between network qualities such as speed, redundancy, accessibility, power use and scalability. Consisting of wires, servers and switches, these physical networks can also be hybrid or, through use of optical as opposed to electronic switches, reconfigure during runtime. On top of these variously designed hardware networks, DCIM software controls the virtualisation process, assigning load to different physical machines and moving virtual machines across them in variable patterns to optimise operations. One reason why this east-west traffic is so important is because it underlies peering relations that allow firms to realise comparative advantages by creating connections with the servers of other firms in the same installation. Peering arrangements enable the elimination of intermediaries and the reduction of transit times for data packets that would otherwise run through external networks such as the Internet. They also allow rapid sharing and agglomeration of data that can train machine learning and other artificial intelligence algorithms. Without understanding the network topologies and virtualisation processes operative within data centres, it is difficult to track or intervene in the application of automated technologies along the server-client axis where labour becomes vulnerable.

Like other technologies of logistical coordination and automation, these developments seek to reduce the turnover time of capital. Such 
efforts are pronounced in high-frequency trading, a financial sector renowned for its requirements of low-latency computing. New routines of remote sensing and edge computing feed the fantasy that the data centre might begin to stand free of the human labour forces that operate at its client end. That millisecond differences are at stake makes it seem as if all this proceeds inevitably and beyond the reach (or at least the speed) of human cognition. As Harney and Moten (2013, p. 87) write: "Logistics wants to dispense with the subject altogether". Ultimately, however, a server cannot eliminate its clients. This is true even if a frontend machine usually needs to consult several other servers in the same data centre before it can send an aggregated response back to a client. To recognise the server's need for clients is not to perpetuate the perversion of the master-slave dialectic or to suggest that clients come first, as twentieth-century corporations once claimed of their customers. Rather, it is to register capital's increased dependence on so-called externalities. In the data economy, capital extracts value from fronts of social cooperation that it does not necessarily control or coordinate in the manner that it once did in the industrial factory.

If, from the viewpoint of media theory, automation presents the growing ubiquity of autonomous systems, from the perspective of capital it creates a scenario of increased reliance on its outsides. Data are the index of this reliance. While the data centre load may register distant labour and thus the continued presence of living knowledge, intelligence and subjectivity, the data generated by this labour, paid or unpaid, present capital with new frontiers of accumulation. In this sense, we cannot immediately equate the extraction of value that fuels data economies with the extraction of surplus value that characterises so-called free wage labour. Recalling Rosa Luxemburg's (1913/2003) argument that accumulation requires a society that consists of more than capitalists and workers, we can register how data provide capital with an outside to prospect and draw upon. To understand data as such an outside is to recognise that they arise from forms of human cooperation and social activity that capital does not necessarily organise, even if they are subject to a kind of projective logic by which capital constructs them as susceptible to appropriation. If, for Luxemburg, writing in the early twentieth century, the outside of capital consisted of different territories that could 
be colonised and integrated into the capitalist world system, the fronts presented by data today are more profuse and topologically variegated. The data centre is the infrastructural facility that sucks in and agglomerates these diverse bodies of data, subjecting them to forms of analysis and extraction that can themselves be automated and serve the ends of further automation at the client interface. The location of data production sites with respect to standard topographies of national political space and international territorial borders remains relevant for legislation and policy. However, such arrangements are increasingly inflected and challenged by topologies of digital connection that support processes of extraction, valorisation and accumulation that national governments struggle to control.

\section{Installing Data}

How then are we to understand the relations between labour, capital and data? In an article entitled Data: The Currency of Tomorrow, Jayson Goh, the former Executive Director of the Infocomms and Media Division of Singapore's Economic Development Board, writes: "Data is a raw resource, just like labour and capital, but one that needs to be farmed, milled and modelled into an effectively usable form" (Goh, 2013 , p. 8). The metaphor of data as a raw resource is dominant in industry and government attempts to make sense of data's relation to labour and capital. Present whenever there is talk of data mining, the implication of the metaphor of raw data is that data are there for the taking, like resources in the ground. Even in the case of resource mining, however, capital and labour are necessary to make the extractive process productive. Tsing (2005) gives the example of the sorting, grading and transportation that are necessary to make a coal supply chain function, beyond the extraction of raw material from the earth. The same applies to data, where the extracted material is not natural resources but patterns of social cooperation. Data, in this sense, are always "cooked" (Gitelman, 2013), regardless of whether they are produced by human input, as in the case of social media, or by social practices and activities detected through remote sensing or other Internet of Things technologies. As Goh 
(2013) recognises, such data must be "farmed, milled and modelled" into a usable or, perhaps better, a fungible form. This work of aggregation, processing and analysis occurs in data centres, where the smoothing out or cleaning of data makes them machine readable and thus ready to train algorithms to perform automated tasks.

However securitised the data centre may be, and whatever the protocols of commercial secrecy that apply to its operations and clientele, such farming, milling and modelling does not take place in a vacuum. Just as automation requires machines to interact with their environments or external conditions of operation, rather than performing a series of mandated tasks in serial patterns, the data centre is an installation that situates itself in diverse material contexts. If environments of automation consist increasingly of data themselves, drawn in from various kinds of sensing technologies, the environments of data centres remain physical as well as computational. These environments are multiple and include the urban milieus to which data centres maintain weak social ties. They also include national environments that partially set regulatory frames for data centre operations and infrastructural environments provided by electrical grids, cable networks and the like. Routines of supply and maintenance furnish hardware environments, while software environments encompass different aspects of code and algorithms. Finally, natural environments supply the energy and water resources for which data centres have a seemingly insatiable thirst.

The load of data centres on natural environments is an important area of social and ecological concern. Research connects data centres to the energy infrastructures on which they depend and, in turn, to the intensification of global warming (see, e.g., Carruth, 2014; Hogan, 2015; Starosielski, 2014). Aware of its environmental footprint, the data centre industry responds by setting its own green standards and handing out awards on their basis. Whatever the impact of data centres on natural environments, and undoubtedly it is significant, the need of these facilities for power and water draws arguments about them back to the fundamentals of agrarian political economy: The dispossession of land, water, forests and other common property is part of the conditions of their existence. However, a mere quantification of the power and water data centres use cannot account for the multiple environments 
in which these installations exist. These environments, importantly, are not merely technical and ecological but also social and cultural. They encompass the urban milieu in which data centres are set, the shifting social relations that result from the communicative and locational media they support, and, more widely, the aesthetic environments that their operations culturally inflect and which, in turn, react back upon public consciousness of these infrastructures. An example of such an aesthetic environment is the spectacle generated by Karl Lagerfeld's transformation of the Grand Palais catwalk into a makeshift data centre for the launch of Chanel's 2017 prêt-à-porter line at Paris Fashion Week (Stinson, 2016).

Not by accident do we refer to data centres as installations. The aesthetic medium of the installation, which is blatant in the Lagerfeld example or in artworks and films that take data centres as a theme, is also evident in the infrastructure and design of data centres themselves. The title of Cisco IT architect Douglas Alger's book The Art of the Data Center (2012) registers how the aestheticisation of the data centre is not merely an act carried out by external agents such as artists or fashion designers but integral to the technological environment of data centre design itself. Another example of this aestheticisation comes in the work of cable technicians responsible for wiring together servers. For all of the standardisation of communication protocols, there is remarkable variation in the aesthetics of cable and wiring configurations twisting across the ports of server racks. Many data centres present uniform cabinets of servers in stark contrast to the chaotic tangle of cables that snake across the hot and cold aisles of patch panels that bring servers into communicable relation. Other installations display a splendour that resembles a cadaver splayed out for the acquisition of scientific skills. Revered as art among technicians, cabling establishes the distributed topology of routers and switches in data centres. What qualifies as art for technicians passes as porn for other kinds of spectators. A prominent aggregator site where these punters share their art features the byline: "A place for sharing links to good-looking cabling (primarily data centres) that could be considered art (or porn)" (Cable Porn; the art of tidy data centres, 2018).

The aesthetics of cable configurations index the spatial distinction of data centre installations on global scales. As one Singapore-based 
company specialised in data centre infrastructure and management solutions touts on their site: "Standard rack configurations won't work well in every data centre. Space constraints, cable routing, power configurations and cooling environments vary" (DC Gears, 2018, n.p.). Not only, then, do external conditions influence the orchestration of cable configurations. These installations also support a range of data centre functions. Whether configured for faster backup and retrieval speeds, scalability for storage, flexibility, security or management considerations, data centre cabling provides an optic of data typologies not easily gleaned from conventional ethnographies of infrastructure.

Regardless of the network topology chosen for a data centre, the large number of switches and servers in these installations means that faults and failures are a regular feature of their operations. Routing mechanisms must make use of redundancies offered by hardware. Not by accident is one of the main industry bodies in the data centre industry known as the Uptime Institute. As Mosco (2014, p. 9) writes: "cloud companies promise and their customers expect, that data centres will operate with no down time". Many data centres run diesel power generators, which are kept turning over so that they can kick in in the event of an external power failure. Typologies of fault tolerance move us into different classifications of data centre failure: failure type, failure region, failure neighbourhood, failure mode and failure time (Liu et al., 2013, pp. 51-53). Failure in the form of "crashes and viruses, bloatware, malware and vaporware", as media theorist Cramer (2005, p. 9) notes, comprises the "irrationality of rational systems".

An aesthetic allure crosses the spectrum of failure. Contingency, like failure, is multihued. A particular state may be, during the course of economic history, notable as an infrastructural centre or growth area for one technological form or another. This does not mean ipso facto that these technological forms harmonise with others in ways that avoid failures of interoperability. Nor does it mean that a dominant technological form generates social harmony within or beyond the state. Singapore's data centres position the island-state as an infrastructural hub. Managers seek to assure system stability and longevity with an eye to not just internal machine operations but also to burgeoning competition in the market throughout the Asian region. More particularly, the materiality of 
data centres as an infrastructural form is spatially extensive and prone to vagaries of failure that do not always conform to those listed in manuals. As we argue in the next section, the effort to introduce standards is a bulwark against failures, and not accidentally has the production of standards for artificial intelligence become a sharp area of geopolitical competition that shapes the contemporary world across its spatial and economic dimensions.

\section{The Automated State}

We earlier suggested that Singapore provides a strategic site to observe the growing competition between China and the US to set artificial intelligence standards because firms from both these states are active in the island's data centre industry. Singapore, for its part, has sought to play a role as an intermediary to Chinese business since the Deng Xiaoping era of opening. More recently, Singapore has opened its doors to Chinese data centre operators such as Aliyun (Alibaba Cloud), Tencent and China Mobile. The Chinese search engine provider Baidu runs an artificial intelligence research centre in the island's Fusionopolis technology park (Apostolou, 2012). In turn, Singapore has invested in data centres in China via the government-linked corporation Singapore Technology Telemedia, which has a stake in twenty-six mainland data centres (Choudhury, 2017). Singapore's sovereign wealth fund Temasek Holdings is an investor in Chinese artificial intelligence startups such as Sensetime, which specialises in surveillance, and Rokid, a robotics firm with voice technology capabilities. While it is true that Chinese firms also set up data centres in neighbouring countries like Malaysia and Thailand, Singapore remains their main launch pad for expansion in Southeast Asia and provides a context where they mingle and compete with US operators such as Google, Amazon and Microsoft. This means Singapore's future as a data centre hub rests largely in the race between China and the US to establish standards for artificial intelligence.

Although China's data protectionist policies have generally been interpreted in relation to the Great Fire Wall and issues of freedom and democracy, they also mean that the country has amassed a great deal 
of data on which to build artificial intelligence. As opposed to nations who opened their doors to US tech giants and have thus had data generated by users in their territories drained out and used to generate services that might potentially be sold back to them, China has extracted its own data and set its own digital industry standards. While this poses business advantages, it also presents challenges when it comes to international expansion, especially as most standards have been set in the US. Perhaps this is why China has prepared a New Generation Artificial Intelligence Development Plan (State Council of China, 2017) and a follow-up White Paper on Artificial Intelligence Standards (Standards Administration of China, 2018) to lay out a strategy for shaping artificial intelligence governance and policy at the international level (Ding et al., 2018). Huawei senior director Wael Diab has become chairperson of the subcommittee of the International Standards Organisation and the International Electrotechnical Commission working on artificial intelligence standards. Not accidentally did this body, formed in 2017, hold its first meeting in Beijing.

While the outcomes of China's effort to shape artificial intelligence standards are unknown, they are important not only for commercial but also geopolitical reasons. China seeks a "right to speak" at the international table of technology development and is also keen to ensure the roll out of automated governance technologies, such as its social credit system, without a huge social backlash, as occurred with India's introduction of its Aadhaar social identity number. China's claims for assuring the safety and social acceptance of artificial intelligence are serious, even if its international expansion in the field is likely to proceed in the absence of the civil society voices that shape the debate in Europe and North America. Playing a key role in standardisation promises to increase the value derived from automated technologies due to data pooling and interoperability. China's intervention also seeks to improve the competitiveness of its firms by working essential patents into protocols and thus obliging other firms to pay royalties when building equipment. With its long-standing practice of piracy and violation of intellectual property rights, China suffered humiliation from signing up to the World Trade Organisation's Agreement on Trade-Related Aspects of Intellectual Property Rights in 2001. Never admitted as a board 
member of the US dominated Internet Corporation for Assigned Names and Numbers (ICANN), the Chinese government maintained its own regulatory processes and engaged ICANN through the organisation's Governmental Advisory Committee. China also played an obstructionist role in the United Nation's World Summit on the Information Society multistakeholder Internet governance meetings held in the early 2000s. Now China intends to exact recompense by building its own artificial intelligence capabilities. Such a project, particularly when it takes the form of standard setting, promises to change the global business environment, giving Chinese technology firms greater leverage to enter new markets, particularly but not only in the developing world.

As the unravelling of neoliberalism brings more countries to favour the models of state capitalism developed in China and Singapore, the relations between states and tech companies are likely to shift. Such changes are particularly relevant in contexts where automation technologies threaten job loss. Call centre jobs in the Philippines, for instance, are currently under threat from the Google automated voice response system Duplex (Gonzales, 2018), which would doubtless be served from its data centre in Singapore. However, the Duplex roll out is delayed due to ethical questions of machines imitating humans (Lomas, 2018), and Chinese firms such as the aforementioned Rokid, financed by Singapore's sovereign wealth fund, are not far behind in developing their own voice recognition chip (Jao, 2018). Chinese influence on technical and ethical standards could open new channels of investment and automation in the Philippine call centre market. Tracing these possible changes is a task for future research. For now, a focus on the territorial and operative dimensions of data centres promises to give a more nuanced picture than either the vision of Morozov (2018), for whom states retain the capacity to rein in tech companies, or Bratton (2015), whose totalising vision of information infrastructures posits a global nomos that eclipses state power. States do not go away, but neither are they singular entities. Morozov (2018) remains wedded to an order of international states, while Bratton's (2015) preference is for a politics of things in which humans drift into aesthetic reverie.

If automation creates autonomous systems, we need to ask how such autonomy measures up in the political sphere. In political theory, 
autonomy registers either as an attribute of sovereignty or as a characteristic of independent political organisation that exists beyond the state. Tech companies market automated services not only to commercial entities but also to political bodies such as states and municipalities. The discourse of the smart city is the most obvious symptom of such efforts, raising the question of how economic returns and political powers of surveillance, control and decision flow to corporations and algorithms rather than to formally constituted political bodies and the citizens, or denizens, that compose them. We think the nexus of automation and sovereignty is more complex than smart city debates allow.

To claim that states retain the ability to control autonomous systems is to underestimate the extent to which automation contributes to governance. The demise of censuses and other state-run statistical surveys in the face of the vast amounts of data held by social media and other tech companies is a register of this. Similar observations apply to other areas of government, including healthcare, taxation, welfare and policing. Today, even military applications run from the securitised environment of commercial data centres. Yet to pretend that infrastructures of automation float free from established political institutions-states, parties, bureaucracies and the like-is to enter a fantasy world where clear cutdivisions between politics and commerce structure the articulation of power. We can certainly recognise data centres as autonomous infrastructures that operate in parallel, rivalry and partnership with states. This vision can take multiple shapes, from Robert MacBride's (1967) call in The Automated State for a national computing centre (in the United States) that would remain independent from any one of the government's arms to Keller Easterling's (2014) more recent and transnationalised mapping of emergent infrastructural powers in Extrastatecraft. But everywhere states are hitting back against the autonomy of data, whether it be through routine protocols of surveillance, firewalls, national nets, policy visions that make "smartness" a principle of nationalism or legislation that mandates so-called data sovereignty.

Data sovereignty measures, in particular, are a front of state paranoia in the face of the proliferation of data. As Chander and Lê (2015, p. 680) explain, there are many ways in which states seek to encumber the transfer of data across national borders. These methods include: 
rules preventing information from being sent outside the country, rules requiring prior consent of the data subject before information is transmitted across national borders, rules requiring copies of information to be stored domestically, and even a tax on the export of data.

Selby (2017, p. 213) argues that laws requiring data to be stored within a particular jurisdiction "are being supported by some countries not only as a means to reduce their comparative disadvantage in internet data hosting, but also to reduce their comparative disadvantage in internet signals intelligence". Whether they understand motives as economic or political, attempts to understand data sovereignty tend to assume a baseline model of the state more or less Weberian in provenance: a territorial entity that holds the legitimate monopoly of violence over a given community. We tend to think, by contrast, that data sovereignty laws represent an effort by states to assert themselves as such an entity within a global political situation that has drastically changed. The sovereignty of media arises at precisely the point the state presumes itself to wield authority within data environments already surrendered to the automation of decision.

Debates about the governmentalisation of the state have questioned its unity. At the same time, critiques of the state's exceptional powers haunt traditional visions of sovereignty. In the world of Marxist theory, it seems no longer possible for the state to act, to control, or mediate the aggregate operations of capital let alone vouchsafe the social reproduction of labour. We are neither enthusiastic for arguments about the withering away of the state nor for reactive positions that proclaim it has never changed. We also recognise, as Gupta (2012, p. 52) puts it, that arguments about the state must always ask the question "which state?" Nonetheless, we must measure the transformations of state power against the operative powers manifest in data centres. To contend that state legislation will bring these powers to heel is to make an assumption about where the seats of power lie these days. The situation, we think, is more open. The decision of a country like Singapore to welcome data centres to its shores by no means registers a dynamic of power where legislation and policy trump infrastructure every time. 


\section{Conclusion}

If data centres crystallise political powers, they are likely to become a crucial point of investigation and intervention for movements that seek to change the world. Although debate has evolved in relation to the nexus of automation and labour, a widening ambit of political and economic analysis is necessary to pose accurate questions about the impact of automation on workforces. This chapter shows that such analysis is not possible in the absence of considerations of media, extraction, standards, geopolitics, territory and infrastructure that expand the ambit of political economy and science and technology studies. If our focus on the data centre industry in Singapore has drawn attention to developments in Southeast Asia, this interest has also been strategic insofar as it raises the issue of how data centres reconfigure world regions and highlights geopolitical struggles. The growing prospect of technological "decoupling" between China and the US is certainly relevant here, although its dynamics have played out somewhat more visibly in relation to $5 \mathrm{G}$ networking systems. What remains the case is that if regions such as Europe hope to establish some autonomy in the data economy, allowing for an escape from a zero sum choice between adopting US or Chinese technologies, the development of data centres within regulatory environments attentive to the ownership and sharing of data that undergird machine learning and automation routines will be key. Our point is that such regulation will not necessarily be delivered by state powers that must already reckon with the political relevance of automated decisionmaking. The prospect to recalibrate technologies of automation in ways that do not serve capital accumulation rests on ongoing struggles and modes of organisation that script new horizons as externalities not yet trafficked through global infrastructures of control. This is the art of installation without the sovereign.

Acknowledgements The Authors acknowledge the financial support by the Australian Research Council (ARC DP160103307). 


\section{References}

Alger, D. (2012). The art of the data center: A look inside the world's most innovative and compelling computing environments. Prentice Hall.

Apostolou, N. (2012, July 27). Baidu opens R\&D lab in Singapore: Chinese answer to Google gets into speech recognition. The Register. https://www. theregister.com/2012/07/27/baidu_opens_first_lab/. Accessed 21 February 2021.

Benanav, A. (2019). Automation and the future of work-2. New Left Review, 120, 117-146.

Bratton, B. (2015). The stack: On software and sovereignty. MIT Press.

BroadGroup. (2016). Data centre South East Asia. BroadGroup.

Cable porn; the art of tidy data centres. (2018). Reddit. https://old.reddit.com/ r/cableporn/. Accessed 21 February 2021.

Carruth, A. (2014). The digital cloud and the micropolitics of energy. Public Culture, 26, 339-364.

Chander, A., \& Lê, U. P. (2015). Data nationalism. Emory Law Journal, 64, 677-739.

Chang, J.-H., \& Hunyh, P. (2016). ASEAN in transformation: The future of jobs at risk of automation (Bureau for Employers' Activities, Working Paper No. 9). International Labour Organization.

Choudhury, A. R. (2017, August 22). STT data centre arm set up for next stage of global expansion. Business Times. https://www.businesstimes.com. $\mathrm{sg} /$ technology/stt-data-centre-arm-set-for-next-stage-of-global-expansion. Accessed 21 February 2021.

Couldry, N., \& Mejias, U. A. (2019). The costs of connection: How data is colonizing human life and appropriating it for capitalism. Stanford University Press.

Cramer, F. (2005). Words made flesh: Code, culture, imagination. Piet Zwart Institute.

DC Gears (2018). Customizable racks. http://www.dcgears.com.sg/racks-enclos ures/racks/customizable-racks. Accessed 21 February 2021.

Ding, J., Triolo, P., \& Sacks, S. (2018). Chinese interests take a big seat at the AI governance table: Government and industry team to shape emerging AI standards-setting process. New America. https://www.newamerica.org/cyb ersecurity-initiative/digichina/blog/chinese-interests-take-big-seat-ai-govern ance-table/. Accessed 21 February 2021.

Easterling, K. (2014). Extrastatecraft: The power of infrastructure space. Verso. 
Fazi, M. B. (2018). Can a machine think (anything new)? Automation beyond simulation. AI \& Society, 34, 813-824.

Frey, C. B., \& Osborne, M. A. (2013). The future of employment: How susceptible are jobs to computerisation? Resource document. University of Oxford. http://www.oxfordmartin.ox.ac.uk/downloads/academic/The_Fut ure_of_Employment.pdf. Accessed 21 February 2021.

Gitelman, L. (Ed.). (2013). "Raw data" is an oxymoron. MIT Press.

Goh, J. (2013). Data: The currency of tomorrow. Resource document. Singapore Economic Development Board. https://www.edb.gov.sg/content/dam/.../ Singapore-Business-News-May-2013.pdf. Accessed 24 October 2018.

Gonzales, G. (2018, July 6). The Google tech that may threaten call centre jobs. Rappler. https://www.rappler.com/technology/features/google-duplexcall-center-philippines-threat. Accessed 21 February 2021.

Gupta, A. (2012). Red tape: Bureaucracy, structural violence and poverty in India. Duke University Press.

Harney, S., \& Moten, F. (2013). The undercommons: Fugitive planning \& black study. Minor Compositions.

Hogan, M. (2015). Facebook data storage centers as the archive's underbelly. Television \& New Media, 16, 3-18.

Innis, H. A. (1951). The bias of communication. University of Toronto Press.

Jao, N. (2018, June 5). Chinese AI startup Rokid will mass produce their own custom AI chip for voice recognition. TechNode. https://technode.com/ 2018/06/05/rokid/. Accessed 21 Feb 2021.

Liu, Y., Muppala, J. K., Veeraraghavan, M., Lin, D., \& Hamdi, M. (2013). Data centre networks: Topologies, architectures and fault-tolerance characteristics. Springer.

Lomas, N. (2018, May 10). Duplex shows Google failing at ethical and creative AI design. TechCrunch. https://techcrunch.com/2018/05/10/duplex-showsgoogle-failing-at-ethical-and-creative-ai-design/. Accessed 21 February 2021.

Luxemburg, R. (1913). Die Akkumulation des Kapitals. Heptagon. English edition: Luxemburg, R. (2003). The accumulation of capital (trans: Schwarzschild, A., foreword: Kowalik, T.). Routledge.

MacBride, R. (1967). The automated state: Computer systems as a new force in society. Chilton.

Méda, D. (2016). The future of work: The meaning and value of work in Europe (ILO Research Paper No. 18). International Labour Organization.

Mezzadra, S., \& Neilson, B. (2017). On the multiple frontiers of extraction: Excavating contemporary capitalism. Cultural Studies, 31, 185-204. 
Miller, S. M. (2018). AI: Augmentation, more so than automation: A managerial perspective on how firms can effectively deploy human minds and intelligent machines in the workplace. Asian Management Insights, 5(1), $1-20$.

Morozov, E. (2018, April 1). After the Facebook scandal it's time to base the digital economy on public $\mathrm{v}$ private ownership of data. The Guardian. https://www.theguardian.com/technology/2018/mar/31/bigdata-lie-exposed-simply-blaming-facebook-wont-fix-reclaim-private-inform ation. Accessed 21 February 2021.

Mosco, V. (2014). To the cloud: Big data in a turbulent world. Paradigm.

Regalado, A. (2012, July 11). When machines do your job. MIT Technology Review. https://www.technologyreview.com/s/428429/when-mac hines-do-your-job/. Accessed 21 February 2021.

Selby, J. (2017). Data localization laws: Trade barriers or legitimate responses to cybersecurity risks, or both? International Journal of Law and Information Technology, 25, 213-232.

Srnicek, N., \& Williams, A. (2016). Inventing the Future: Postcapitalism and a World without Work. Verso.

Standards Administration of China (2018). White paper on artificial intelligence standardization. Resource document. http://www.sgic.gov.cn/upload/ f1ca3511-05f2-43a0-8235-eeb0934db8c7/20180122/5371516606048992. pdf. Accessed 24 October 2018.

Starosielski, N. (2014). The materiality of media heat. International Journal of Communication, 8, 2504-2508.

State Council of China (2017). New generation of artificial intelligence development planning. http://www.gov.cn/zhengce/content/2017-07/ 20/content_5211996.htm. Accessed 24 October 2018.

Stinson, E. (2016, April 10). Chanel turned its fashion show runway into a data centre. Wired. https://www.wired.com/2016/10/chanel-turnedfashion-show-runway-data-center/\#: : :text=Chanel\%20Turned $\% 20$ Its $\%$ 20Fashion\%20Show $\% 20$ Runway\%20Into $\% 20$ a,colorful\%20ethernet $\%$ 20 cords $\% 20$ spewed $\% 20$ from $\% 20$ stacks $\% 20$ of $\% 20$ servers. Accessed 23 February 2021.

Sverdlik, Y. (2017, February 13). How to get a data centre job at Google. Data Centre Knowledge. https://www.datacenterknowledge.com/archives/ 2017/02/13/how-to-get-a-data-center-job-at-google. Accessed 23 February 2021.

Tsing, A. (2005). Friction: An ethnography of global connection. Princeton University Press. 
Wajcman, J. (2017). Automation: Is it really different this time? The British Journal of Sociology, 68, 119-127.

Open Access This chapter is licensed under the terms of the Creative Commons Attribution 4.0 International License (http://creativecommons.org/ licenses/by/4.0/), which permits use, sharing, adaptation, distribution and reproduction in any medium or format, as long as you give appropriate credit to the original author(s) and the source, provide a link to the Creative Commons license and indicate if changes were made.

The images or other third party material in this chapter are included in the chapter's Creative Commons license, unless indicated otherwise in a credit line to the material. If material is not included in the chapter's Creative Commons license and your intended use is not permitted by statutory regulation or exceeds the permitted use, you will need to obtain permission directly from the copyright holder.

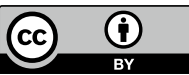

